\title{
Interactions and correlations of particulate inclusions in a columnar phase
}

\author{
Ronojoy Adhikari \\ Centre for Condensed Matter Theory, Department of Physics, \\ Indian Institute of Science, Bangalore 560 012, India
}

(Dated: June 21, 2021)

\begin{abstract}
We calculate the elastic field mediated interaction between macroscopic particles in a columnar hexagonal phase. The interaction is found to be long-ranged and non-central, with both attractive and repulsive parts. We show how the interaction modifies the particle correlations and the column fluctuations. We also calculate the interaction of particles with the topological defects of the columnar phase. The particle-defect interaction reduces the mobility of the defects.

PACS numbers: $61.30 . \mathrm{Cz}, 82.70 . \mathrm{Dd}$
\end{abstract}

\section{INTRODUCTION}

Macroscopic particles suspended in an isotropic fluid colloidal dispersions - have been the subject of intensive study for over a century [1]. Recently, a novel class of materials have been realised in which colloidal particles are dispersed in anisotropic fluids, typically thermotropic and lyotropic liquid crystals [2, 3, 4. The combination of anisotropy and long-range order inherent in these mesophases leads to totally new kinds of interactions between the dispersed particles, which have no analogues in conventional colloidal dispersions [5, 6, 7, 8, 97.

The most important of these new interactions results from the elastic deformation induced by a particle in the liquid crystalline host. The overlap of the the elastic deformations of two particles produces an effective long-ranged interaction between them. This mechanism, which relies only on the mean distortions of the order parameter, implies that other sources of deformation like topological defects also interact with particles through long-range forces. Fluctuations of the order parameter together with the boundary conditions on the particle surface lead to another novel kind of Casimir-like interaction [10], which can often be important near special points in the phase diagram. In particle-doped nematics, for instance, director fluctuations lead to a Casimir force which is stronger than the van der Waals force near the isotropic-to-nematic transition [9]. Interparticle interactions are further complicated by the inevitable presence of topological defects surrounding the particles [8]. These defects compensate the mismatch in the boundary conditions imposed on the order parameter at the particle surface and at infinity. To clarify with an example, a particle in a nematic with director-normal boundary conditions on its surface is equivalent to a radial hedgehog with unit positive topological "charge", and is incompatible with the requirement of an uniform director configuration at infinity. The mismatch is relieved by the spontaneous appearance of a defect of unit negative "charge", which is tightly bound to the particle. Such companion de-

*Electronic address: rjoy@physics.iisc.ernet.in fects usually lead to rapidly decaying, repulsive, power law forces between the particles [8]. The interactions mentioned above, when combined with the multitude of interactions already present in conventional colloidal dispersions $[\mathbb{1}$, can drive the particles into spatially organised structures - chains 8, 13], clusters [11, and even crystalline arrangements 12 have been observed for particles in nematic hosts.

As a result of these novel interactions, the static and dynamic properties of (with an obvious extension of terminology) liquid crystalline colloids are quite unusual. The change in the mechanical properties is particularly dramatic - particles added in volume fractions as low as several percent can totally alter the rheological behaviour of the host phase [3, 4, 14]. When the particles have a magnetic moment interesting ferro-liquid crystalline phases are formed [15, 16].

Numerous theoretical studies have been done on these and related effects in nematic colloids [17. Comparatively less work has been done on particle-doped lamellar phases 18, 19, 20, 21]. It is therefore surprising that in spite of recent experiments [15, 16], there has been no theoretical study of the properties of particle-doped columnar phases. In the following sections of this paper, therefore, as a first step towards a more detailed theory, we calculate the elastic field mediated interparticle interactions (section III), as well as the interactions of particles with point topological defects (section III). We conclude the paper with a critical discussion of our results and possible extensions of the present work.

\section{PARTICLES IN A COLUMNAR HEXAGONAL PHASE}

Let us recall that the columnar phase is macroscopically equivalent to a regular array of tubes, aligned in, say, the $z$ direction (directions in the $x-y$ plane are denoted as $\perp$ ) and free to slide along their axes. A cross-sectional slice normal to the tubes shows a twodimensional crystal structure, which, for the case we study, is hexagonal. Mechanically, the phase is liquidlike in the $z$ direction, and solid-like and isotropic in the $\perp$ directions. Elastic deformations are described by a 
pair of "broken-symmetry" variables $\mathbf{u}=\left(u_{x}, u_{y}\right)$ corresponding to the local displacements of the tubes from their equilibrium position. To lowest order in gradients, the harmonic free energy density includes contributions from in-plane bulk and shear deformations, and out-ofplane column curvatures [23]:

$$
\begin{aligned}
\mathcal{F}_{\text {hex }} & =\frac{B}{2}\left(\nabla_{\perp} \cdot \mathbf{u}\right)^{2}+\frac{K_{3}}{2}\left(\nabla_{z}^{2} \mathbf{u}\right)^{2} \\
& +\frac{C}{2}\left[\left(\nabla_{x} u_{x}-\nabla_{y} u_{y}\right)^{2}+\left(\nabla_{y} u_{x}+\nabla_{x} u_{y}\right)^{2}\right]
\end{aligned}
$$

Here $B$ and $C$ are first-order elastic constants (with dimensions of energy density) corresponding to bulk and shear deformations respectively, while $K_{3}$ is a secondorder elastic constant (with the dimension of force) corresponding to column curvature. An elastic description of this sort is valid at length scales large compared to the column spacing $d$. The colloidal particles are modelled as monodisperse spheres of radius $a$ and are described by a concentration field $c(\mathbf{r})=\sum_{\nu} \delta\left(\mathbf{r}-\mathbf{r}_{\nu}\right)$, where $\mathbf{r}_{\nu}$ is the center-of-mass coordinate of the $\nu$ th particle. A description of this sort is valid at length scales large compared to the particle radius $a$, when in effect, the particles can be treated as point masses. In this dilute limit, where the typical distance between particles is much larger than their size, all short-range interactions can be ignored, and the particles need to be assigned only an ideal gas entropy. As in the theory of point defects in three dimensional crystals, it is assumed that the particles behave as force dipoles ("centers of dilation or compression") 24] and produce a body force proportional to $\nabla c(\mathbf{r})$ at the point $\left(\mathbf{x}_{\perp}, z\right)=\mathbf{r}$. This is equivalent to a coupling of the form $c(\mathbf{r}) E(\mathbf{u})$ in the free energy, where $E(\mathbf{u})=\nabla_{\perp} \cdot \mathbf{u}$ is the local dilation. It is not difficult to see that to linear order in $\mathbf{u}$ and lowest order in gradients, this is the only possible symmetry-allowed coupling of the concentration to the column displacement [25]. With these considerations, then, the free energy density (in units of $k_{B} T$ ) is

$$
\mathcal{F}(c, \mathbf{u})=\mathcal{F}_{h e x}+c(\mathbf{r})\left[\ln \frac{c(\mathbf{r})}{c_{0}}-1\right]+\alpha c(\mathbf{r}) E(\mathbf{u}) .
$$

The second term in (2) accounts for the entropy of the particles whose mean concentration is $c_{0} . \alpha$ is a coupling constant, which on dimensional grounds must be of order $B a^{3}$. Note that in our units $B$ has the dimensions of inverse volume and that $\alpha$ is dimensionless. The microscopic details of the boundary conditions on the particle surface are incorporated phenomenologically in the sign and magnitude of $\alpha$. For positive $\alpha$ the columns are repelled away from the particle surface leading to a local dilation; this situation is most appropriate for inert colloidal particles, whose size $a$ is appreciably larger than the column spacing $d$. For negative $\alpha$ the columns are are attracted towards the particle leading to a local compression; this situation may be appropriate for small biological inclusions, which are known to exert local inward forces on their surroundings [19, 39]. Henceforth, we shall assume $\alpha$ to be positive. It is worth emphasising that our theory is valid for separations large compared to both $d$ and $a$, which represent, respectively, the scales beyond which the elastic and concentration field descriptions are meaningful.

\section{A. Effective Interactions}

Integrating out the displacement field $\mathbf{u}$ from $F[c, \mathbf{u}]=$ $\int d^{3} \mathbf{r} \mathcal{F}(c, \mathbf{u})$ we obtain an effective free energy

$$
F_{c}=-\ln \left(\int \mathcal{D} \mathbf{u} e^{-F[c, \mathbf{u}]}\right)
$$

for the concentration fluctuations. Since our interest lies only in the mean displacements of the columns, the Gaussian integral in (3) can instead be replaced by the minimum of $F[c, \mathbf{u}]$ over $\mathbf{u}$. Setting the variation $\delta F / \delta \mathbf{u}$ to zero, we obtain a pair of coupled linear differential equations for the components $\left(u_{x}, u_{y}\right)$. Transforming to Fourier space (ie multiplying by $e^{-i \mathbf{q} \cdot \mathbf{r}}$ and integrating over $d^{3} \mathbf{r}$ ), and solving the resulting algebraic equation for the Fourier components, we have

$$
\mathbf{u}_{\mathbf{q}}=\frac{i \alpha \mathbf{q}_{\perp}}{(B+C) q_{\perp}^{2}+K_{3} q_{z}^{4}} c_{\mathbf{q}} .
$$

This equation gives the (mean) displacement field for an imposed concentration fluctuation $c_{\mathbf{q}}$. Using it to eliminate the displacement field from (2), we obtain the effective free energy as

$$
F_{c}=\int d^{3} \mathbf{r} c(\mathbf{r})\left[\ln \frac{c(\mathbf{r})}{c_{0}}-1\right]+\frac{1}{2} \int_{\mathbf{q}} c_{\mathbf{q}} V(\mathbf{q}) c_{-\mathbf{q}}
$$

where

$$
V(\mathbf{q})=\frac{-\alpha^{2} q_{\perp}^{2}}{(B+C) q_{\perp}^{2}+K_{3} q_{z}^{4}}
$$

and we have used the shorthand $\int_{\mathbf{q}} \equiv \int \frac{d^{3} \mathbf{q}}{(2 \pi)^{3}}$. The second term in (5) represents the effective interaction mediated by the column displacements. The kernel $V(\mathbf{q})$ can be identified with the Fourier transform of the interparticle potential $V(\mathbf{r})$, with the understanding that the correct physics is captured only for lengths large compared to the particle size. With that in mind, the interparticle potential is given by

$$
\begin{aligned}
V(\mathbf{r}) & =\frac{\alpha^{2} \lambda_{3}^{2}}{K_{3}} \nabla_{\perp}^{2} G\left(\mathbf{x}_{\perp}, z\right) \\
& =\frac{\alpha^{2} \lambda_{3}^{2}}{K_{3}} \nabla_{\perp}^{2} \int_{0}^{\infty} d t \frac{e^{-z^{2} / 4 \lambda_{3} x_{\perp} \cosh t}}{\left(\lambda_{3} x_{\perp} \cosh t\right)^{1 / 2}}
\end{aligned}
$$

where $\lambda_{3}^{2}=K_{3} /(B+C)$. The Fourier transform $G\left(\mathbf{x}_{\perp}, z\right)$ of the kernel $\left(q_{\perp}^{2}+\lambda_{3}^{2} q_{z}^{4}\right)^{-1}$ can be obtained explicitly in 


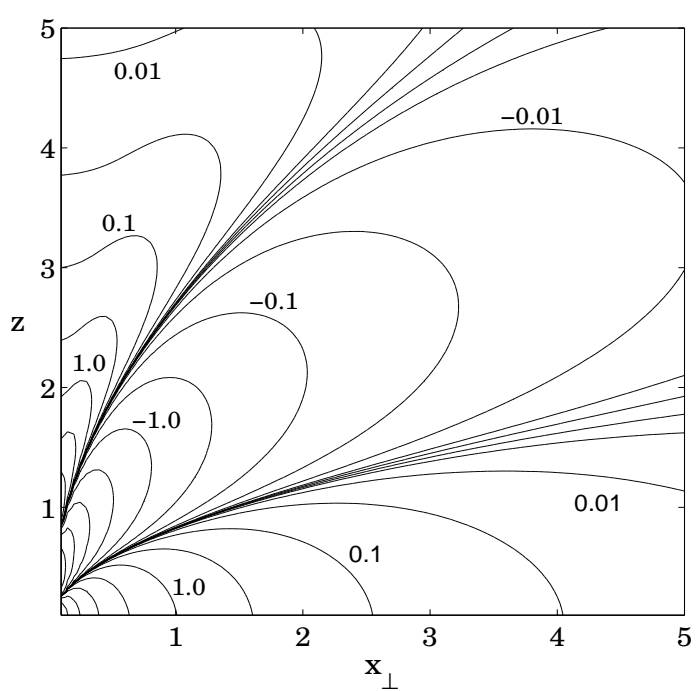

FIG. 1: Plot of the equipotentials of $V(\mathbf{r}) \sim \nabla_{\perp}^{2} G(\mathbf{r})$. The coordinates are in units of $\lambda_{3}$. The contour lines have been drawn on equal logarithmically spaced intervals from .001 to 1000 , and -0.001 to -1000 . The potential is even in $z$.

terms of modified Bessel functions [29], but we prefer the integral representation given above.

The potential $V(\mathbf{r})$ presents several interesting features. It is non-central (not a function of $r$ only) and radially non-monotonic. This last property prevents us from inferring the sign of the radial component of the force from that of the potential. Therefore, the attractive or repulsive character of the interaction cannot be correlated with the sign of the potential, and the forces have to be considered explicitly. However, in the $z$ and $\perp$ directions the potential is monotonic and decays with a power law. Near the $z$-axis (for $z^{2} \gg 4 \lambda_{3} x_{\perp}$ ) the potential vanishes as $|z|^{-5}$, leading to a rapidly damped and repulsive force along the columns.Near the $x-y$ plane (for $z^{2} \ll 4 \lambda_{3} x_{\perp}$ ) the potential decays more slowly as $x_{\perp}^{-5 / 2}$, implying a repulsive force in the $\perp$ direction. In a general direction the nature of the interaction is best visualised through the plot of the equipotentials of $V(\mathbf{r})$ shown in Fig.11. From the plot it is clear that the radial component of the force is positive near the $z$-axis and the $x-y$ plane, and negative in the region bounded by the two paraboloids $z^{2} \sim \lambda x_{\perp}$. Broadly speaking, the force felt by a particle at $\left(\mathbf{x}_{\perp}, z\right)$ due to another particle at the origin varies from repulsive, to attractive, to repulsive again as the polar angle $\theta=\tan ^{-1}\left(z / x_{\perp}\right)$ varies from 0 to $\pi / 2$. Since the potential is even in $z$, the sequence repeats as the angle decreases from $\pi$ to $\pi / 2$. A qualitatively similar angular dependence is also found in the quadrupolar forces between particles in a nematic [6]; the distance dependence, though, is totally different.

Given the nature of the interparticle forces described above, we might expect an attraction induced aggregation of the particles. However, repulsive forces due to the companion topological defects mentioned in the intro- duction may dominate at short distances, and arrest the aggregation. In that case, it is likely that particles will spontaneously organise in chains oblique to the columns. This process will be dominated by Brownian motion, and not the elastic interaction, which though long-ranged, decays too rapidly to affect the kinetics of aggregation.

The correction to the interparticle potential due to a finite density of particles may be calculated quite easily within a mean field approximation [26]. Augmenting the free energy $F_{c}$ with an external potential $V_{e x t}(\mathbf{r})$ which couples to the concentration, the equilibrium concentration is obtained by demanding $\delta F_{c} / \delta c=0$. This gives

$$
c(\mathbf{r})=c_{0} \exp \left[-V_{e x t}(\mathbf{r})-\int d^{3} \mathbf{r}^{\prime} c\left(\mathbf{r}^{\prime}\right) V\left(\mathbf{r}-\mathbf{r}^{\prime}\right)\right] .
$$

The mean (or effective) potential is defined by $c(\mathbf{r})=$ $c_{0} e^{-U(\mathbf{r})}$. Using this to eliminate the density from the previous equation and identifying the external potential with that of a fixed particle at the origin, we obtain the following self-consistent equation for $U(\mathbf{r})$ :

$$
U(\mathbf{r})=V(\mathbf{r})+c_{0} \int d^{3} \mathbf{r}^{\prime} V\left(\mathbf{r}-\mathbf{r}^{\prime}\right) \exp \left[-U\left(\mathbf{r}^{\prime}\right)\right] .
$$

Assuming that $U \ll 1$ we can linearise the above integral equation. Neglecting the term corresponding to the background concentration and Fourier transforming we find

$$
U(\mathbf{q})=\frac{V(\mathbf{q})}{1+c_{0} V(\mathbf{q})}=\frac{-\alpha^{2} q_{\perp}^{2}}{\left(B+C-c_{0} \alpha^{2}\right) q_{\perp}^{2}+K_{3} q_{z}^{4}} .
$$

The mean potential is of the same functional form as the "bare" interparticle potential, but with a "renormalised" characteristic length $\lambda_{3}^{2}(\alpha)=K_{3} /\left(B+C-c_{0} \alpha^{2}\right)$. Recalling that a similar calculation with a Coulomb potential $V(\mathbf{q}) \sim q^{-2}$ leads to charge screening [26], we must conclude that, despite the long-ranged interaction, manyparticle effects are quite modest in our case.

\section{B. Correlation Functions}

In the absence of interactions the particles in our model have only configurational entropy and their twopoint correlations are like those of an ideal gas: < $\delta c(\mathbf{r}) \delta c\left(\mathbf{r}^{\prime}\right)>\sim \delta\left(\mathbf{r}-\mathbf{r}^{\prime}\right)$. However, elastic interactions lead to quite complex correlations, as we show below. Defining the dimensionless structure factor $S(\mathbf{q})$ as the Fourier transform of $\frac{1}{c_{0}}<\delta c(\mathbf{r}) \delta c\left(\mathbf{r}^{\prime}\right)>$ we find from the effective free energy in (5) that

$$
\begin{aligned}
S(\mathbf{q}) & =\frac{1}{1+c_{0} V(\mathbf{q})} \\
& =1+\frac{c_{0} \alpha^{2} q_{\perp}^{2}}{\left(B+C-c_{0} \alpha^{2}\right) q_{\perp}^{2}+K_{3} q_{z}^{4}} .
\end{aligned}
$$

The Fourier transform of the pair correlation function $h(\mathbf{q})=S(\mathbf{q})-1$ is proportional to $-U(\mathbf{q})$, and therefore 
the pair correlations themselves are well described by the contour plot of Fig.11 with all the signs reversed. The correlations are negative along the $z$ and $\perp$ axes, indicating a reduction in the particle concentration compared to the mean value of $c_{0}$, while in the region bounded by the paraboloids $z^{2} \sim \lambda_{3} x_{\perp}$ the correlations are positive, indicating an enhancement of the concentration. This is broadly consistent with our earlier picture of particle chains oblique to the columns. It may be possible to measure the particle structure factor by small-angle Xray scattering, and the form of $S(\mathbf{q})$ above would then provide a test of the particle-column coupling assumed in our theory.

While the column displacements induce particle correlations, the particle aggregates in turn modify the column fluctuations. In particle-doped lamellar phases, this feedback mechanism has been shown to change the usual Caille [37] form of the structure factor [21]. To calculate the column displacement correlations in the presence of particles, we first define the displacement field such that $\left\langle\nabla_{i} u_{j}\right\rangle=0$ for a mean concentration $c_{0}$. This requires that we modify the particle-column coupling to $\delta c(\mathbf{r}) E(\mathbf{u})$. Next, we define an effective free energy $F_{\mathbf{u}}$ for the column fluctuations (in a way similar to the definition of $F_{c}$ ) by integrating out the concentration field. Carrying out the integration by steepest descent we obtain the effective free energy

$$
F_{\mathbf{u}}=\int d^{3} \mathbf{r}\left[\mathcal{F}_{h e x}-c_{0}\left(\alpha \nabla_{\perp} \cdot \mathbf{u}-e^{-\alpha \nabla_{\perp} \cdot \mathbf{u}}\right)\right]
$$

This shows that the coefficients of all symmetry-allowed terms which contain $\nabla_{\perp} \cdot \mathbf{u}$ are "renormalised" by the presence of particles. Within our harmonic description the only relevant term, obtained by expanding the exponential term to quadratic order, is $\alpha^{2}\left(\nabla_{\perp} \cdot \mathbf{u}\right)^{2}$. Retaining only this term in (12) and defining the longitudinal and transverse parts of $\mathbf{u}_{\mathbf{q}}$ as $q^{2} \mathbf{u}_{L}=\mathbf{q}\left(\mathbf{q} \cdot \mathbf{u}_{\mathbf{q}}\right)$ and $\mathbf{u}_{T}=\mathbf{u}_{\mathbf{q}}-\mathbf{u}_{L}$ we obtain the $u_{L(T)}-u_{L(T)}$ correlations as

$$
\begin{gathered}
\left|\mathbf{u}_{T}\right|^{2}=\frac{1}{C q_{\perp}^{2}+K_{3} q_{z}^{4}}, \\
\left|\mathbf{u}_{L}\right|^{2}=\frac{1}{\left(B+C-c_{0} \alpha^{2}\right) q_{\perp}^{2}+K_{3} q_{z}^{4}} .
\end{gathered}
$$

The transverse correlations are identical to those of the pure columnar phase, which indicates that there are no corrections to $C$ and $K_{3}$ due to the particles. The longitudinal correlations while identical in form, have a modified bulk modulus $B_{c}=B-c_{0} \alpha^{2}$. Particles thus produce a softening of the columnar phase.

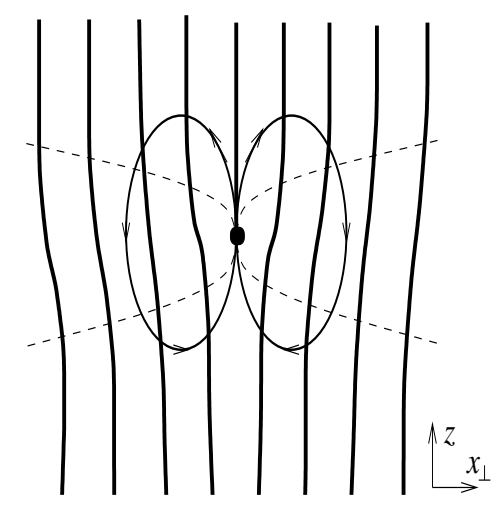

FIG. 2: A "tail" defect configuration of topological charge $m=+1$ with the thick lines showing the column deformations column near the defect. The particle-defect potential vanishes on the paraboloids $z^{2} \sim \lambda_{3} x_{\perp}$ (dashed lines), beyond which it is mainly repulsive for $z>0$ and attractive for $z<0$. The thin lines show typical trajectories of overdamped particles: they are expelled from the regions of dilation $(z>0)$ and attracted to the regions of compression $(z<0)$.

\section{PARTICLE-DEFECT INTERACTIONS}

\section{A. Point defects in the columnar phase}

Topological defects are common in liquid crystalline phases, being easily produced under conditions of processing such as shear flows.Much of the interesting macroscopic behaviour of liquid crystalline colloids comes from the interaction of the colloidal particles with the topological defects of the host [3, 4, 14. For example, the solid-like response of particle-doped cholesteric [3] and smectic [1] phases is due to the formation of a stable network of defects. The colloidal particles play the crucial role of stabilising this network, which otherwise would anneal away. It is likely that elastic interactions do not play a dominant role in such processes, and that shortrange forces, not captured in a coarse-grained description such as ours, are more important. Nevertheless, elastic interactions between particles and topological defects are known to have important consequences [36], and so in what follows, we calculate this interaction for a point defect configuration in the columnar phase. The statistical mechanics of thermally activated point defects [27, 35] may be modified in the presence of particles and a knowledge of particle-defect interactions could be potentially useful in a study of this phenomenon. An interesting duality (see below) between the columnar and lamellar phases has been noted by de Gennes and Prost [23] and explored in detail by Selinger and Bruinsma [28]. This suggests that it may be of interest to compare particledefect interactions in the lamellar and columnar phases and we conclude this section with such a comparison.

The defect configuration we consider is a single column ending 28, 29], illustrated in Fig. 22. This is not unduly restrictive, since any linear defect which consists 
of column endings (eg. a transverse edge dislocation) can be constructed out of a collection of such point defects [29]. The ease with which a column can break depends on what constitutes the "column", and therefore, on what the particular realisation of the columnar phase is. In polymeric columnar phases, the columns consist of long chains arranged end-to-end along their length. Here, thermally activated column scission can occur only at those places where one end of a chain meets that of another. (Polymeric liquid crystals are found predominantly in the nematic and isotropic phases; however, longer and stiffer biopolymers like DNA can also positionally order into columnar phases [31]). Since columns in discotic and micellar phases are composed of weakly bonded molecules, thermally activated scission can take place anywhere along the length of the columns. In all of these examples, the column scission energy is finite and thus a state of thermal equilibrium at non-zero temperature will always contains a finite number of such defects. The ends of a scissioned column may either separate along the $z$-axis to form a string of vacancies, or may translate past each other in the $\perp$ direction to form a string of interstitials [27]. Such strings will begin from and end on the column extremities, the lower of which, by convention, is called a "tail" (Fig. 2) and the upper a "head" [27]. At high temperatures the entropy of wandering of the string can overcome the energy due to its line tension, leading to a string proliferation, and producing isolated "heads" or "tails" [29, 30]. Signatures of such defects have been observed in recent experiments [32]. An isolated semi-infinite string running along the $z$-axis with its terminus at the origin imposes the constraint that 28]

$$
\nabla_{\perp} \cdot \mathbf{u}=m \frac{\delta\left(\mathbf{x}_{\perp}\right)}{\rho_{\mathbf{0}}} \theta(z)
$$

where the topological "charge" $m$ is $+1(-1)$ for a tail (head), and $\rho_{0}$ is the areal density of the columns. Clearly this is nothing but a line of centers of dilation and is easily treated in the framework of the previous section. Formally, a column terminus at the origin is identical to a line of point particles distributed with the concentration

$$
c(\mathbf{r})=m \frac{(B+C)}{\alpha \rho_{0}} \delta\left(\mathbf{x}_{\perp}\right) \theta(z) .
$$

Combining this with the expression in (伍) for the displacement produced by a distribution of particles, the displacement due to a column ending is obtained as 33.

$$
\mathbf{u}_{\mathbf{q}}=\frac{i \mathbf{q}_{\perp}}{q_{\perp}^{2}+\lambda_{3}^{2} q_{z}^{4}} \frac{m}{\rho_{0} i q_{z}} .
$$

The displacement for a collection of column endings distributed with the topological "charge" density $m(\mathbf{r})$ follows from the superposition of the individual displacements. Stated mathematically, this means that the total

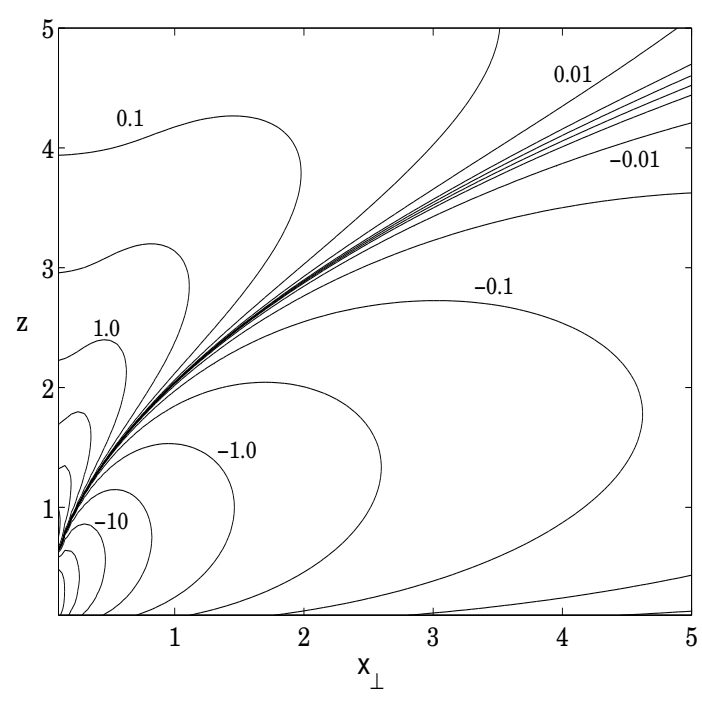

FIG. 3: Plot of the equipotentials of $V_{c m}(\mathbf{r}) \sim \nabla_{z}^{3} G(\mathbf{r})$. The coordinates are in units of $\lambda_{3}$.The contour lines have been drawn on equal logarithmically spaced intervals from .001 to 100 , and -0.001 to -100 . The potential is odd in $z$.

displacement is

$$
\mathbf{u}_{\mathbf{q}}=\frac{i \mathbf{q}_{\perp}}{q_{\perp}^{2}+\lambda_{3}^{2} q_{z}^{4}} \frac{m_{\mathbf{q}}}{\rho_{0} i q_{z}},
$$

with $m_{\mathbf{q}}$ being the Fourier transform of $m(\mathbf{r})$. To calculate the particle-defect interaction, the displacement field is decomposed as $\mathbf{u}_{\mathbf{q}}=\mathbf{u}_{\mathbf{q}}\left(c_{\mathbf{q}}\right)+\mathbf{u}_{\mathbf{q}}\left(m_{\mathbf{q}}\right)$, where the first part (due to the particles) is obtained from (4) and the second part (due to the defects) is obtained from (18). The free energy then separates out as $F=F_{c}+F_{m m}+F_{c m}$, with

$$
F_{m m}=\frac{1}{2} \int_{\mathbf{q}} \frac{q_{\perp}^{2}}{\rho_{0}^{2} q_{z}^{2}} \frac{m_{\mathbf{q}} m_{-\mathbf{q}}}{q_{\perp}^{2}+\lambda_{3}^{2} q_{z}^{4}} \frac{K_{3}}{\lambda_{3}^{2}}
$$

and

$$
F_{c m}=\int_{\mathbf{q}} \frac{i \alpha q_{\perp}^{2}}{\rho_{0} q_{z}} \frac{m_{\mathbf{q}} c_{-\mathbf{q}}}{q_{\perp}^{2}+\lambda_{3}^{2} q_{z}^{4}} .
$$

The Fourier transforms of the defect-defect and particledefect interactions can now be read off as the kernels of $m_{\mathbf{q}} m_{-\mathbf{q}}$ and $c_{\mathbf{q}} m_{-\mathbf{q}}$ in (19) and (20) respectively. The transformation to real space can be simplified by noting that

$$
\nabla_{\perp}^{2} G\left(\mathbf{x}_{\perp}, z\right)=\lambda_{3}^{2} \nabla_{z}^{4} G\left(\mathbf{x}_{\perp}, z\right) .
$$

Using this property, $q_{\perp}^{2}$ can be replaced by $\lambda_{3}^{2} q_{z}^{4}$ in the numerators of the kernels in $(19)$ and $(20)$. Alternatively, a conjugate Green's function can be introduced as in 28] and 35]. With either of the methods, the final form of the interaction kernels turn out to be

$$
V_{m m}(\mathbf{q})=\frac{K_{3}}{\rho_{0}^{2}} \frac{q_{z}^{2}}{q_{\perp}^{2}+\lambda_{3}^{2} q_{z}^{4}},
$$


and

$$
V_{c m}(\mathbf{q})=\frac{\alpha \lambda_{3}^{2}}{\rho_{0}} \frac{i q_{z}^{3}}{q_{\perp}^{2}+\lambda_{3}^{2} q_{z}^{4}} .
$$

Apart from the line tension terms which we ignore, the defect-defect interaction is identical to the result of [28] and has been discussed in detail there [34]. The particledefect interaction can be represented in real space as

$$
V_{c m}(\mathbf{r})=\frac{m \alpha \lambda_{3}^{2}}{\rho_{0}} \nabla_{z}^{3} G\left(\mathbf{x}_{\perp}, z\right) .
$$

As might have been anticipated, the particle-defect potential is non-central and radially non-monotonic, and is odd in the $z$-coordinate. The behaviour in the $z$ and $\perp$ directions is simple. Along the columns (for $z^{2} \gg 4 \lambda_{3} x_{\perp}$ ) the potential decays as $\operatorname{sgn}(z)|z|^{-4}$ indicating a repulsive force for $z>0$ and an attractive force for $z<0$. In the $x$ - $y$ plane, the $\perp$ component of the force vanishes by symmetry and the $z$ component goes to zero as $x_{\perp}^{-5 / 2}$. The detailed form of the interaction for positive $z$ is shown in the equipotential plot of Fig.3. The general conclusion is that independent of the sign of the defect, the particles are attracted to the regions of compression, and repelled away from the regions of dilation that the defect alone would have produced, see Fig.2. Driven by the attractive component of the interaction, and aided by Brownian motion, a cloud of particles should collect around a column ending, a situation reminiscent of the Cottrell clouds of vacancies and interstitials around dislocations in metals [36]. Normally, such clouds decrease the dislocation mobility [36] and the same can be assumed to happen here. Since defect motion is one of the main mechanisms of stress relaxation in ordered phases, less mobile defect strings may lead to an increase in the viscosity of the columnar phase.

It is important to note that expression (18) can be used to obtain the interaction of a particle with any linear defect which can be represented as a collection of column endings. For example, the interaction of a transverse edge dislocation with a particle can be obtained from (18) by setting $m(\mathbf{r}) \sim \delta(y) \delta(z)$ and repeating the steps leading to (20).

\section{B. Line defects in the lamellar phase}

There is an interesting line-plane duality [23, 28] between defect-free columnar and lamellar phases which is apparent in a pictorial representation: a set of lines representing the columns (aligned, say, along the $z$-axis) when rotated by $\pi / 2$ can equally well be viewed as a set of planes representing the lamellar layers (now stacked normal to the $z$-axis). Mechanically, the lamellar phase is a $2 \mathrm{~d}$ liquid and a $1 \mathrm{~d}$ solid, while the columnar phase is a $1 \mathrm{~d}$ liquid and a $2 \mathrm{~d}$ solid. Though this duality is lost in the presence of an arbitrary ensemble of defects (for example screw dislocations), nonetheless there is one important exception. It is easy to see from Fig. 2 that an endpoint defect (the terminus of a line) in the columnar phase corresponds to an edge dislocation (the terminus of a plane) in the lamellar phase. Going beyond pictures, there are close connections in the energetics of these "dual" defects, which have been explored in [28. This prompts us to ask if similar connections may be found in the interactions of endpoints and edge dislocations with particles. To answer this question, we first examine the particle-dislocation interaction for an arbitrary distribution of dislocations, and then specialise to the case of an edge dislocation.

The free energy density of a particle-doped lamellar phase where the particles couple to the local layer dilation has been obtained in [19]. Excluding the entropic terms, in our notation it is

$$
F=\frac{\bar{B}}{2} \int d^{3} \mathbf{r}\left[\left(\nabla_{z} u\right)^{2}+\lambda^{2}\left(\nabla_{\perp}^{2} u\right)^{2}+\bar{\alpha} c(\mathbf{r}) \nabla_{z} u\right],
$$

where $u$ is the local displacement of the layers from their equilibrium position, $B$ is the bulk modulus, $K=B \lambda^{2}$ is a second order elastic constant corresponding to layer curvature, and the positive constant $\bar{\alpha}$ is the strength of the particle-layer coupling. In the presence of a dislocation density $\mathbf{b}(\mathbf{r})$ the distortion $\mathbf{v}=\nabla u$ acquires a singular transverse part determined by [18]

$$
\nabla \times \mathbf{v}=\mathbf{b}(\mathbf{r})
$$

The total distortion in Fourier space is given by 26]

$$
\mathbf{v}_{\mathbf{q}}\left(\mathbf{b}_{\mathbf{q}}\right)=\frac{i \mathbf{q} \times \mathbf{b}_{\mathbf{q}}}{q^{2}}+i \mathbf{q} h_{\mathbf{q}}
$$

where $h_{\mathbf{q}}$, the longitudinal part of the distortion, is obtained from the equation of equilibrium $\delta F / \delta u=0$. The solution

$$
h_{\mathbf{q}}=\frac{\bar{\alpha} i q_{z} c_{\mathbf{q}}}{q_{z}^{2}+\lambda^{2} q_{\perp}^{4}}-\frac{q_{z}\left(1-\lambda^{2} q_{\perp}^{2}\right)}{q_{z}^{2}+\lambda^{2} q_{\perp}^{4}} \frac{\left(\hat{\mathbf{z}} \cdot \mathbf{q} \times \mathbf{b}_{\mathbf{q}}\right)}{q^{2}}
$$

is a sum of the particle and defect contributions. When the distortion in (27) is inserted into the (Fourier transformed) free energy (28), the latter separates into three pieces: the first contains only the dislocation density [26, the second only the concentrations [19], and the third is a cross-term which represents the particle-dislocation interaction. Displaying only this last term, we have

$$
F_{c b}=\bar{B} \int_{\mathbf{q}} \frac{\bar{\alpha} \lambda^{2} q_{\perp}^{2}}{q_{z}^{2}+\lambda^{2} q_{\perp}^{4}}\left(\hat{\mathbf{z}} \cdot \mathbf{q} \times \mathbf{b}_{\mathbf{q}}\right) c_{-\mathbf{q}} .
$$

This gives the interaction energy of an arbitrary distribution of particles with an arbitrary number of dislocations. Two important special cases are linear edge and screw dislocation of infinite extent. The dislocation density for a screw dislocation of infinite extent and Burgers vector $b \hat{\mathbf{z}}$ is $\mathbf{b}(\mathbf{r})=b \delta(x) \delta(y) \hat{\mathbf{z}}$, which implies that 


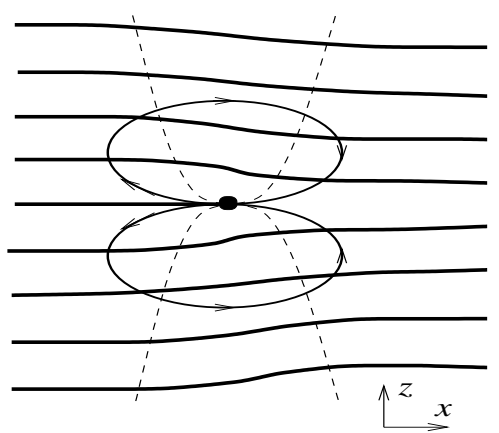

FIG. 4: An infinite edge dislocation of Burgers charge $b=+1$ running along the $y$-axis with the thick lines showing the layer deformations near the defect. The dashed lines show the parabolas $x^{2}=4 \lambda|z|$ for arbitrary values of $y$. The thin lines show typical trajectories of overdamped particles: they are expelled from the regions of dilation $(x<0)$ and attracted to the regions of compression $(x>0)$. Notice that this configuration is obtained by rotating Fig. 2 through $\pi / 2$.

$\mathbf{b}_{\mathbf{q}}=2 \pi b \delta\left(q_{z}\right) \hat{\mathbf{z}}$. Inserting this into $(29)$ we find that there is no elastic interaction between a particle and the screw component of a dislocation within a linear elastic theory. For the case of interest, an infinite edge dislocation running along the $y$-axis with Burgers vector $b \hat{\mathbf{z}}$, $\mathbf{b}(\mathbf{r})=b \delta(x) \delta(z) \hat{\mathbf{y}}$, and $\mathbf{b}_{\mathbf{q}}=2 \pi b \delta\left(q_{z}\right) \hat{\mathbf{z}}$, and so together with (29) the particle-edge dislocation interaction kernel is obtained as

$$
V_{c b}(\mathbf{q})=\frac{\bar{B} \bar{\alpha} \lambda^{2} q_{\perp}^{2}}{q_{z}^{2}+\lambda^{2} q_{\perp}^{4}} 2 \pi b i q_{x} \delta\left(q_{y}\right) .
$$

Transforming to real space, the interaction potential is

$$
V_{c b}(\mathbf{r})=\frac{\bar{B} \bar{\alpha} b}{8} \frac{x}{\sqrt{\pi \lambda|z|^{3}}} \exp \left(\frac{-x^{2}}{4 \lambda|z|}\right) .
$$

The interaction is long-ranged along the $z$-axis (for $x^{2} \ll 4 \lambda|z|$ ) and exponentially damped along $x$-axis (for $\left.x^{2} \gg 4 \lambda|z|\right)$. By symmetry, the $z$-component of the force vanishes on the $z$-axis, while both the $x$ and $z$ components are exponentially small near the $x$-axis. Irrespective of the sign of $b$, the particles are attracted to the compression regions that the dislocation alone would have produced, and repelled away from the dilation regions, see Fig.(化). To compare this interaction with its dual in the columnar phase, we first note that the interaction is characterised by the parabolas $x^{2}=4 \lambda|z|$ which occur frequently in problems related to dislocations in lamellar phases [23]. The particle-defect potential in the columnar phase is characterised instead by the paraboloids $z^{2} \sim \lambda_{3} x_{\perp}$. In both cases the maximum elastic deformation is confined to the regions within these surfaces, compare Fig.(2) and Fig.(14). The force is long-ranged along the solid-like directions (the $\perp$ direction in the columnar phase, and the $z$ direction in the lamellar phase) and short-ranged in the liquid-like directions. There is no interaction between a particle and an edge dislocation when both have the same $z$ coordinate, a feature also seen in the interaction between two edge dislocations [23]. In contrast, the particle-defect interaction in the columnar phase is strongly repulsive in the $z$ direction. The formation of Cottrell clouds, and the consequent reduction in the dislocation mobility should be expected in the lamellar phase as well.

\section{CONCLUSION}

To summarise, we have calculated the elastic interactions of colloidal particles in a columnar hexagonal phase by modelling them as centers of dilation. The interaction is long-ranged and non-central, and has both attractive and repulsive parts. We have shown that this interaction leads to correlations between the particle positions, and to a reduction in the bulk modulus $B$ of the columnar phase. We have also shown how particles interact elastically with defects in columnar and lamellar phases. In both phases, particles aggregate around the defects driven by the attractive component of the interaction and reduce the defect mobility. One likely consequence of this is an increase in the viscosity of the phase.

We mention below several of the directions in which the present work may be extended. First, the elastic interactions of particles of more general shapes like cylinders and discs should be examined. Such inclusions can no longer be modelled as force dipoles, and force distributions containing higher multipoles will be needed [24. The interaction of plate-like particles would have a direct relevance to the physics of clay-block copolymer composites [22]. Second, the effects of boundaries should be investigated. Free boundaries require the introduction of image sources, which will have long-range interactions with particles in the bulk. Thus, boundary effects can be expected to strongly influence all processes in the bulk, and in particular, the formation of particle aggregates. Third, the nature of the topological defects surrounding a particle should be examined. Our coarse-grained description cannot predict the defect configuration (though useful information can be gained if we assume it) and a more microscopic description would be better suited for the purpose.

As a final, somewhat speculative remark, we should indicate that the present calculation may be of some relevance in biological systems where inclusions may be present in tissues of columnar hexagonal structure [38. Biological inclusions generate local inward forces 19, 39. and a minimal description of such inclusions could be in terms of the centers of compression (corresponding to a negative $\alpha$ ) in our theory. Similar elastic interactions between proteins in a lamellar phase [20], and cells in an isotropically elastic tissue have already been investigated 39. 


\section{Acknowledgments}

The author would like to thank Professor Sriram Ramaswamy for suggesting the problem, useful discussions, and a critical reading of the manuscript, and Professor Chandan Dasgupta for helpful suggestions. Partial support from Hindustan Lever Limited is gratefully acknowledged.
[1] W. B. Russel, D. A. Saville, W. R. Schowalter, Colloidal Dispersions (Cambridge Univ. Press, Cambridge,1989)

[2] P. Poulin, V. A. Raghunathan, P. Richetti, D. Roux, J.Physique II 4, 1557 (1994).

[3] M. Zapotocky, L. Ramos, P. Poulin, T. C. Lubensky, D. A. Weitz, Science,283, 209 (1999).

[4] G. Basappa, Suneel, V. Kumaran, P. R. Nott, S. Ramaswamy, V. M. Naik, D. Rout, Eur. Phys. Jour. B 12, 269 (1999).

[5] F. Brochard, P.-G. de Gennes, J. Physique 31, 691 (1970).

[6] S. Ramaswamy, R. Nityananda, V. A. Raghunathan, J. Prost, Mol. Cryst. Liq. Cryst. 288, 175 (1996).

[7] R. W. Ruhwandl, E. M. Terentjev, Phys. Rev. E 55, 2958 (1997).

[8] P. Poulin, H. Stark, T. C. Lubensky, D. A. Weitz, Science 275, 1770 (1997)

[9] D. Bartolo, D. Long, J.-B. Fournier. Europhys. Lett 49, 729 (2000).

[10] M. Kardar, R. Golestanian, Rev. Mod. Phys 71, 1233 (1999).

[11] V. A. Raghunathan, P. Richetti, D. Roux, Langmuir 12, 3789 (1996).

[12] V. G. Nazarenko, A. B. Nych, B. I. Lev, Phys. Rev. Lett 87, 075504 (2001).

[13] P. E. Cladis, M. Kleman, P. Pieranski, C. R. Acad. Sc. Sér. B 273, 275 (1971). The context of this experiment is somewhat different from ours, since the authors observe chaining of liquid crystalline bubbles at a fluid interface.

[14] S. P. Meeker, W. C. K. Poon, E. Terentjev, J. Crain, Phys. Rev. E 61, R6083 (2000).

[15] L. Ramos, P. Fabre, R. Ober, Eur. Phys. J. B 1, 319 (1998).

[16] L. Ramos, P. Fabre, L. Fruchter, Eur. Phys. J. B 8,67 (1999)

[17] R. W. Ruhwandl, E. M. Terentjev, in Modern Aspects of Colloid Dispersions, R. Ottewill, A. Rennie eds. (Kluwer, 1998)

[18] P. S. Pershan, J. Prost, J. Appl. Phys. 46, 2343 (1975)

[19] M. S. Turner, P. Sens, Phys. Rev. E 55, 1275 (1997)
[20] M. S. Turner, P. Sens, P. Pincus, Phys. Rev. E 55, 4394 (1997)

[21] P. Sens, M. S. Turner, Eur. Phys. J. E 4, 115 (2001)

[22] J. Groenewold, G. H. Fredrickson, Eur. Phys. J. E 5, 171 (2001)

[23] P.-G. de Gennes, J. Prost, The Physics of Liquid Crystals, 2nd edition (Oxford University Press, New York, 1993).

[24] J. D. Eshelby, in Solid State Physics Vol. 3, F. Seitz, D. Turnbull, eds. (Academic, 1956).

[25] Strictly speaking, the correct rotationally invariant form of the coupling is obtained only if we use the nonlinear dilation $E(\mathbf{u})=\nabla_{\perp} \cdot \mathbf{u}-\frac{1}{2} \nabla_{i} \mathbf{u} \cdot \nabla_{i} \mathbf{u}$.

[26] P. Chaikin, T. Lubensky, Principles of Condensed Matter Physics (Cambridge University Press, 1995).

[27] S. Jain, D. R. Nelson, Phys. Rev. E 61, 1599 (2000).

[28] J. V. Selinger, R. F. Bruinsma, J. Physique II 2, 1215 (1992).

[29] J. Prost, Liq. Cryst. 8, 123 (1990).

[30] E. Frey, D. R. Nelson, D. S. Fisher, Phys. Rev. B 49, 9723 (1994).

[31] R. Podgornik, D. C. Rau, V. Parsegian, Macromolecules 22, 1780 (1989); S. A. Safran, L. A. Turkevich, P. Pincus, J. Phys. Lett (Paris) 45 L-69 (1984)

[32] P. A. Albouy, D. Guillon, B. Heinrich, A. M. Levelut, J. Malthete, J. Physique II 5, 1617 (1995).

[33] Prost 29] has obtained a similar result by treating the column ending as a screw dislocation dipole.

[34] Note that the authors' interpretation of the sign of the defect-defect potential is not correct. See the discussion following our equation (日).

[35] J. Toner, D. R. Nelson Phys. Rev. B 23, 316 (1981).

[36] A. Kosevich, in Dislocations in Solids Vol.1, F.Naborro, ed. (North Holland, 1979).

[37] A. Caillé, C. R. Acad. Sci. Paris B 274, 981 (1972).

[38] Y. Bouligand, in Physics of Defects, Les Houches Lecture Notes, Session XXXV (North-Holland, 1981)

[39] U. S. Schwarz, S. A. Safran, Phys. Rev. Lett 88, 048102 (2002) 